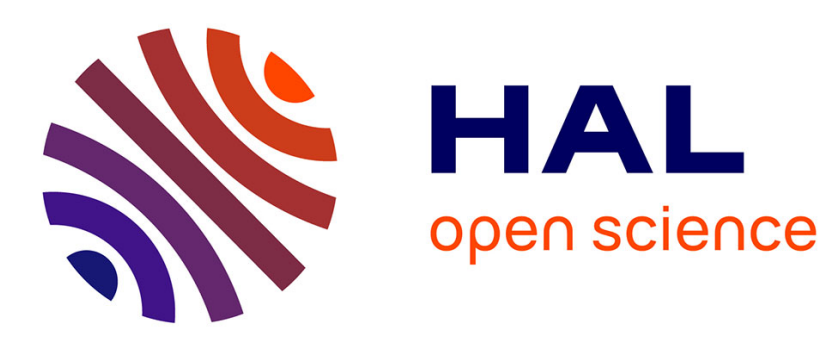

\title{
Une propriété mathématique de la perspective synthétique réfutant son existence médiévale (1295-1450)
}

Dominique Raynaud

\section{To cite this version:}

Dominique Raynaud. Une propriété mathématique de la perspective synthétique réfutant son existence médiévale (1295-1450). Zeitschrift fur Kunstgeschichte, 2004, 67 (4), pp.449-460. halshs-00005700

\section{HAL Id: halshs-00005700 \\ https://shs.hal.science/halshs-00005700}

Submitted on 16 Nov 2005

HAL is a multi-disciplinary open access archive for the deposit and dissemination of scientific research documents, whether they are published or not. The documents may come from teaching and research institutions in France or abroad, or from public or private research centers.
L'archive ouverte pluridisciplinaire HAL, est destinée au dépôt et à la diffusion de documents scientifiques de niveau recherche, publiés ou non, émanant des établissements d'enseignement et de recherche français ou étrangers, des laboratoires publics ou privés. 
Slightly revised for Zeitschrift für Kunstgeschichte, 2004, 67(4) : 449-460

\title{
Une propriété mathématique de la perspective synthétique réfutant son existence médiévale (1295-1450)
}

\author{
Dominique RAYNAUD ${ }^{1}$
}

\begin{abstract}
Résumé : Panofsky et White ont soutenu l'hypothèse que les « constructions axiales » résultent d'un système projectif dans lequel les mesures de l'objet sont prises sur un cercle de projection avant d'être reportées sur un tableau plan (i.e. perspective synthétique). Bien que cette construction ait suscité des critiques quant à la capacité des peintres de la mettre en oeuvre, la thèse d'un usage de la perspective synthétique, jamais étudiée en détail, resurgit régulièrement. Or, ce système de représentation est impossible du point de vue mathématique : dans une perspective synthétique, trois fuyantes prises d'un même côté de l'axe ne peuvent ni être parallèles, ni admettre de point de concours. La réfutation de la conjecture Panofsky-White met un terme à une longue série de jugements contradictoires sur ce problème et ouvre la voie d'une interprétation radicalement différente des constructions axiales.
\end{abstract}

Mots-clefs : perspective linéaire, perspective synthétique, Moyen Âge.

Summary: Panofsky and White hypothezised that « axial constructions » result from a projective system in which object's measurements are taken on an projection circle, before being moved to the picture plane (i.e. synthetic perspective). Although this construction has been yet criticized regarding painter's ability to apply it, the idea of using synthetic perspective regularly reappears for it never has been examined in detail. But from a mathematical point of view, this system is simply impossible because, in a synthetic perspective, three vanishing lines taken on the same side of the axis cannot be parallel nor admit a convergence point. The falsification of Panofsky-White's conjecture puts and end to a long series of contradictory judgements on this problem, and opens the way to a radical reinterpretation of axial constructions.

Keywords: linear perspective, synthetic perspective, Middle Ages.

\section{Introduction}

Avant d'adopter les principes de la perspective linéaire à la Renaissance, les peintres et les architectes ont expérimenté de multiples systèmes de représentation. Certaines oeuvres du Moyen Âge ont été établies selon les principes de la perspective cavalière (qui n'a d'ailleurs de perspective que le nom), ou selon diverses techniques axonométriques. Certains novateurs, comme Giotto, ont recouru concurremment à différents systèmes de représentation. Par exemple, aux fresques de la basilique supérieure d'Assise, ${ }^{2}$ Giotto et son atelier ont utilisé, de 1296 à 1305, une perspective cavalière pour L'Extase, une perspective cavalière " éclatée » pour La Mort du chevalier de Celano, une perspective cavalière doublement éclatée pour La Guéri- 
son du blessé de Léride, mais aussi une dimétrie pour La Fondation des Clarisses, une perspective centrale pour L'Apparition à Grégoire IX et une perspective à deux points ${ }^{3}$ pour Le Prêche à Honorius III. Cet échantillon, pris dans un même atelier, suffit à montrer la diversité des techniques de représentation qui ont été utilisées avant que les peintres n'accordent finalement leur préférence à la perspective linéaire. On doit donc, dans l'étude des oeuvres de cette époque, procéder par division et s'attacher à l'étude d'une famille homogène de représentations - fut-elle hétérodoxe relativement aux canons de la perspective renaissante.

Parmi ces modes de représentation pré-perspectifs (antérieurs à la codification renaissante), on reconnaît généralement l'existence d'un système de « construction axiale », dit encore « en arête de poisson ». Guido Kern, ${ }^{4}$ qui en a fait la découverte, est à l'origine d'une longue trad ition de recherche sur l'existence d'une alternative curviligne dans la représentation perspective. Selon l'auteur, les peintres seraient progressivement passés d'une construction axiale à fuyantes parallèles à une construction axiale à fuyantes convergentes, avant de concevoir un schéma où les fuyantes convergent en un seul point de fuite (perspective linéaire centrale).

Les travaux d'Erwin Panofsky ${ }^{5}$ se caractérisent par une extension de la base empirique des oeuvres suivant le principe de la construction axiale et par la proposition d'une hypothèse permettant leur reconstruction. Ce type de construction aurait déjà été utilisé dans les peintures pariétales de l'Antiquité romaine des $3^{\mathrm{e}}$ et $4^{\mathrm{e}}$ styles de Pompéi ou de Boscoreale $\left(\mathrm{I}^{\mathrm{er}}\right.$ siècle de notre ère), mais l'auteur admet, après Kern, que l'usage de la construction axiale serait surtout une caractéristique du Moyen Âge. Ainsi écrit-il, à propos d'une oeuvre du XIVe siècle, que « les perpendiculaires des secteurs latéraux du plafond courent [...] parallèlement aux consoles qui divisent les plafonds, soit une construction en axe de fuite à l'état pur $»{ }^{6}$ Quant à la r econstruction proposée par Panofsky, elle se fonde sur un court (et énigmatique) passage du traité de Vitruve, dans lequel il est dit que :

« La scénographie est le tracé de la face et des côtés latéraux et le renvoi de toutes les lignes au centre du cercle. ${ }^{7}$ »

Erwin Panofsky - influencé par les recherches sur la perspective curviligne de la fin du $\mathrm{XIX}^{\mathrm{e}}$ siècle $^{8}$ - en déduit que les peintres de l'Antiquité et du Moyen Âge auraient pris la mesure des objets à représenter sur un tableau curviligne, encore nommé « cercle de projection » dont le centre correspond à l'oeil du spectateur. Il applique cette hypothèse à la repré- 
sentation d'une « boîte à espace » (Raumkast), dont on peut reproduire les étapes de construction (Figure 1).

Cette hypothèse de construction géométrique a été reprise par de nombreux historiens de l'art, notamment Little, Bunim et White. ${ }^{9}$

Little $^{10}$ a appuyé les idées de Panofsky en soutenant que la peinture romaine recèle de nombreux cas où le schéma en axe de fuite a une structure géométrique claire. Le caractère géométrique du tracé des fuyantes vaudrait démonstration que cette construction a réellement été appliquée par les peintres de cette époque. Cette idée a été combattue par ceux qui reconnaissent dans de telles oeuvres la marque exclusive d'un tracé empirique.

Miriam Bunim, qui admet certaines objections adressées à cette hypothèse, ${ }^{11}$ accorde toutefois un crédit à l'idée d'un usage généralisé de la construction axiale :

«Les arguments de Panofsky contre l'hypothèse d'une application d'un système de perspective focale dans la peinture antique [...], qu'il dérive de la théorie de l'optique, sont extrêmement convaincants. Le fait que l'on ait remarqué que le champ visuel était sphérique, que l'on ait dit que la taille des objets était proportionnelle à l'angle visuel plutôt qu'à la distance à l'oeil [Euclide, Optica, théor. 8], et que l'on ait observé que les lignes droites subissaient des distorsions curvilignes, tout cela tend à discréditer la possibilité d'un système de perspective focale, car ce sont là les facteurs mêmes qui ne sont pas pris en compte dans cette méthode [...]. L'usage systématique de l'axe de fuite répond au besoin de trouver une méthode perspective satisfaisante qui donne une illusion optique convaincante de l'espace tridimensionnel en même temps qu'elle promeut l'unité de composition de la scène..$^{12}$ »

John White ${ }^{13}$ a également étudié ces constructions axiales. L’un de ses principaux apports est d'appeler ces vues « perspectives synthétiques » ${ }^{14}$ afin d'éviter toute confusion entre con struction axiale et perspective curviligne. En effet, quoique la reconstruction de Panofsky suppose la manipulation d'un tableau curviligne, la représentation obtenue en appliquant cette méthode n'est pas une perspective curviligne dans laquelle les droites doivent être rendues par des grands arcs de cercle. ${ }^{15}$ L'hypothèse de Panofsky se réfère donc à un système mixte, dans lequel les mesures angulaires sont prises sur un tableau curviligne (Figure 1, A-B) avant d'être reportées sur le tableau plan (Figure 1, G). Cette précision d'ordre terminologique n'empêche pas White de se placer dans le sillage direct de Panofsky quant à l'usage de ce système de représentation et quant à l'interprétation qu'il en donne. 
C'est à ces travaux, ${ }^{16}$ concordants quant à l'existence d'une alternative curviligne dans la représentation de l'espace au Moyen Âge, que nous nous référerons désormais sous le nom de « conjecture Panofsky-White ».

Quoique cette conjecture ait suscité les réticences de plusieurs historiens, ${ }^{17}$ l'hypothèse resurgit périodiquement dans le cercle de ceux qui s’intéressent aux problèmes de la représentation médiévale, sans doute parce que Panofsky est une figure emblématique des recherches sur la perspective. J'en veux pour preuve deux exemples récents. Dans un article portant sur la reconstruction ex post de tableaux du XIVe siècle, de Mesa Gisbert parvient à la conclusion que la construction à partir d'un cercle de projection constitue un procédé « impensable, tant pour l'Antiquité que pour le Moyen Âge $»{ }^{18}$ Six ans plus tard, en 1995, Ballardini pense au contraire que les vues de White confirment « [...] l'intelligence perceptive et la précision spéculative par laquelle l'auteur a mis en évidence les relations existant entre la théorie perspective et ses différentes applications au Moyen Âge ». ${ }^{19}$ Placés devant des opinions aussi contr adictoires, il faut avancer des arguments nouveaux, et si possible définitifs, dans le débat.

Quiconque s'est un jour intéressé au problème des constructions axiales sait combien il est difficile d'évaluer la correspondance des tracés à la conjecture Panofsky-White. Panofsky luimême, devant la difficulté à identifier des tracés répondant exactement à son hypothèse, admet l'usage «schématique, mais plus commode, du parallélisme plus ou moins strict des lignes de fuite obliques ». ${ }^{20} \mathrm{Si}$ l'on suit cette recommandation, il existerait en fait deux types de construction répondant au principe de la perspective synthétique : 1) des représentations dans lesquelles on observe un parallélisme des fuyantes ; 2) des représentations dans lesquelles on observe la convergence des fuyantes.

Le parallélisme est un concept géométrique clair. Et certaines oeuvres du Moyen Âge, comme le Retable du Maître de Sainte Cécile, répondent exactement à la condition de parallélisme des fuyantes (Planche 1).

La convergence n'est pas aussi bien définie : elle peut correspondre à des faits différents dès qu'elle concerne plus de trois fuyantes. On peut distinguer deux cas. La convergence stricto sensu signifie que les fuyantes aa', bb', cc'... admettent un unique point de concours M. La convergence lato sensu signifie que ces fuyantes admettent deux à deux des points de concours : aa' et bb' se croisant en M, bb' et cc' se croisant en N, etc. Notons qu'il est plus simple de construire un faisceau de fuyantes répondant à la notion de convergence stricte, car, une fois le point de concours défini, toutes les fuyantes passent par ce point. Le tracé des épures de 
nombreuses perspectives axiales du Moyen Âge indique précisément le type de convergence utilisée par les peintres. Dans toutes les oeuvres du corpus examiné, ${ }^{21}$ les fuyantes, prises d'un même côté du tableau, admettent un point de concours au-delà de l'axe de fuite. Cette propriété, fort simple, n’a pas été remarquée par les historiens de l'art, qui en sont généralement restés à l'opinion de Kern ${ }^{22}$ selon laquelle les fuyantes convergent deux à deux sur l'« axe de fuite ». Il suffit pourtant de prolonger les fuyantes au-delà de l'axe pour faire apparaître les points de concours. Considérons, à titre d'illustration, le cas de fésus parmi les Docteurs de Giusto de' Menabuoi (1376-1378), où les fuyantes concourent en F et F' (Planche 2). L'hypothèse de Panofsky-White devrait rendre compte de toutes les constructions, où les fuyantes issues de la partie gauche admettent un point de concours à droite, où les fuyantes issues de la partie droite admettent un point de concours à gauche.

Cet article vise à mettre un terme à la longue série d'appréciations contradictoires mentionnées plus haut en établissant l'impossibilité mathématique de ce système de représentation. En démontrant que l'hypothèse Panofsky-White n'autorise, ni le parallélisme des fuyantes, ni la convergence des fuyantes en un point de concours, on indiquera que les oeuvres, tenues pour une illustration de la perspective synthétique, doivent être rattachées à un principe de construction perspective inédit.

\section{Propriété de la perspective synthétique}

Reprenons le mode de construction de Panofsky en faisant correspondre la vue perspective et le géométral qui la sous-tend (Figure 2). La boîte à espace étant supposée symétrique, il suffit d'examiner les fuyantes situées à droite de l'axe de vision OK, O étant l'oeil du spectateur. Étant donné que deux droites non parallèles admettent toujours un point de concours, il convient d'étudier trois fuyantes au moins. Considérons donc la partie droite de la boîte $\mathrm{ABEF}$, composée de trois parois $\mathrm{AB}, \mathrm{CD}, \mathrm{EF}$ parallèles à l'axe de vision $\mathrm{OK}$. Un point donné de cette boîte - disons C - intercepte le tableau curviligne IJ, nommé par Panofsky « cercle de projection », en un point $c_{1}$, lequel a pour projection, sur le tableau plan $G H$, le point $c_{2}$. Le rabattement du tableau plan GH donne la vue perspective proprement dite, sur laquelle la droite RS figure l'arête antérieure supérieure BF de la boîte, et sur laquelle la droite PQ est l'image de l'arête postérieure supérieure $\mathrm{AE}$ de la boîte. Le point $\mathrm{C}$ appartenant à $\mathrm{AE}$, la ligne 
de report issue de $c_{1}$ intercepte un point c appartenant à PQ. Nous avons adopté une notation homogène : le quadrilatère abef est l’image de la face supérieure de la boîte ABEF.

Examinons les conditions de convergence ou de parallélisme des fuyantes. Si les fuyantes ba, dc, fe... sont strictement convergentes (admettent un unique point de concours $\mathbf{M}$ ) ou sont parallèles entre elles, le théorème de Thalès prescrit :

$$
\text { [1] } \quad \frac{\mathrm{ac}}{\mathrm{ae}}=\frac{\mathrm{bd}}{\mathrm{bf}}
$$

Les cas du parallélisme et de la convergence des fuyantes répondent à la même propriété. Les segments ac et bd, ae et bf sont proportionnels, avec $\mathrm{df}=$ ce (parallélisme) ou $\mathrm{df} \neq$ ce (convergence).

Cas de la perspective linéaire. Dans une perspective linéaire, les lignes CO, DO, EO, FO sont directement interceptées par le tableau plan GH (Figure 3). Il s'ensuit que la relation [1] équivaut à :

[2] $\frac{\mathrm{Kc}_{1}}{\mathrm{Ke}_{1}}=\frac{\mathrm{Kd}_{1}}{\mathrm{Kf}_{1}}$

Comme $\mathrm{OAC}$ et $\mathrm{OKc}_{1}$ sont des triangles semblables :

[3] $\frac{\mathrm{Kc}_{1}}{\mathrm{OK}}=\frac{\mathrm{AC}}{\mathrm{OA}}$

De même : $\frac{\mathrm{Ke}_{1}}{\mathrm{OK}}=\frac{\mathrm{AE}}{\mathrm{OA}}, \frac{\mathrm{Kf}_{1}}{\mathrm{OK}}=\frac{\mathrm{BF}}{\mathrm{OB}}, \frac{\mathrm{Kd}_{1}}{\mathrm{OK}}=\frac{\mathrm{BD}}{\mathrm{OB}}$.

La relation [2] peut donc s'écrire :

[4] $\frac{\mathrm{AC} / \mathrm{OA}}{\mathrm{AE} / \mathrm{OA}}=\frac{\mathrm{BD} / \mathrm{OB}}{\mathrm{BF} / \mathrm{OB}}$

Soit, après simplification :

[5] $\frac{\mathrm{AC}}{\mathrm{AE}}=\frac{\mathrm{BD}}{\mathrm{BF}}$ 
Ce qui est toujours vrai, puisque $\mathrm{AC}=\mathrm{BD}$ et $\mathrm{AE}=\mathrm{BF}$, par définition. Cela signifie que dans une perspective linéaire centrale, les lignes de fuite correspondant aux droites de bout de l'objet convergent toutes vers un unique point de fuite $\mathrm{M}$.

Cas de la perspective synthétique. Pour que cette propriété soit vraie d'une perspective synthétique, il faudrait que les fuyantes ba, dc, fe... satisfassent une relation équivalente. Ici, les lignes $\mathrm{CO}, \mathrm{DO}, \mathrm{EO}, \mathrm{FO}$ sont interceptées par le tableau curviligne IJ avant d'être reportées sur le tableau plan GH (Figure 2). On a donc les relations :

[6] $\frac{\mathrm{AC}}{\mathrm{AE}}=\frac{\tan \mathrm{AOC}}{\tan \mathrm{AOE}}$ et $\frac{\mathrm{ac}}{\mathrm{ae}}=\frac{\mathrm{Kc}_{2}}{\mathrm{Ke}_{2}}=\frac{\sin \mathrm{AOC}}{\sin \mathrm{AOE}}$

Comme $\tan \alpha=\sin \alpha / \cos \alpha$, il vient :

[7] $\frac{\mathrm{AC}}{\mathrm{AE}}=\frac{\mathrm{ac}}{\mathrm{ae}} \cdot \frac{\cos \mathrm{AOE}}{\cos \mathrm{AOC}}$

De même :

[8] $\frac{\mathrm{BD}}{\mathrm{BF}}=\frac{\mathrm{bd}}{\mathrm{bf}} \cdot \frac{\cos \mathrm{BOF}}{\cos \mathrm{BOD}}$

Comme $\mathrm{AC}=\mathrm{BD}$ et $\mathrm{AE}=\mathrm{BF}$, on a toujours $\frac{\mathrm{AC}}{\mathrm{AE}}=\frac{\mathrm{BD}}{\mathrm{BF}}$, d'où :

[9] $\frac{\mathrm{ac}}{\mathrm{ae}} \cdot \frac{\cos \mathrm{AOE}}{\cos \mathrm{AOC}}=\frac{\mathrm{bd}}{\mathrm{bf}} \cdot \frac{\cos \mathrm{BOF}}{\cos \mathrm{BOD}}$

L'existence d'un point de concours $\mathrm{M}$ devrait se traduire :

$[10] \quad \frac{\mathrm{ac}}{\mathrm{ae}}=\frac{\mathrm{bd}}{\mathrm{bf}}$

Ce qui implique :

[11] $\frac{\cos \mathrm{AOE}}{\cos \mathrm{AOC}}=\frac{\cos \mathrm{BOF}}{\cos \mathrm{BOD}}$ 
Mais comme $\mathrm{OAE}$ et $\mathrm{Oe}_{0} \mathrm{e}_{1}$ sont des triangles semblables, on peut écrire :

[12] $\mathrm{OE}=\frac{\mathrm{OA}}{\cos \mathrm{AOE}}$

Pour les mêmes raisons, on a :

[13] $\mathrm{OC}=\frac{\mathrm{OA}}{\cos \mathrm{AOC}}, \quad \mathrm{OF}=\frac{\mathrm{OB}}{\cos \mathrm{BOF}}, \quad \mathrm{OD}=\frac{\mathrm{OB}}{\cos \mathrm{BOD}}$

Il s'ensuit que la relation [11] équivaut à :

[14] $\frac{\mathrm{OE}}{\mathrm{OC}}=\frac{\mathrm{OF}}{\mathrm{OD}}$ (Figure 2).

Or, cette relation n'est pas toujours vraie. ${ }^{23}$ Contrairement au cas de la perspective l inéaire, la condition $[1 \equiv 14]$ n'est en général pas vérifiée pour une perspective synthétique. Elle ne vaut que dans des cas particuliers qui doivent être examinés.

\section{Étude des solutions particulières}

Les paramètres d'une perspective synthétique que l'on peut faire varier pour rechercher les solutions particulières de la condition [14] sont : la largeur de l'objet, la profondeur de l'objet et la distance du spectateur à l'objet. On peut en outre fixer l'échelle du géométral en posant $\mathrm{AC}=\mathrm{BD}=1$. Introduisons les notations :

$$
\text { [15] } \begin{aligned}
x & =\mathrm{CE} \\
y & =\mathrm{AB} \\
z & =\mathrm{BO} \\
1 & =\mathrm{AC}
\end{aligned}
$$

Par raison de symétrie, on peut limiter l'étude à $x \geq 0, y \geq 0, z \geq 0$.

Supposons que la largeur $x=\mathrm{CE}$ soit variable (Figure 4). On observe que, à mesure que EF s'éloigne de CD, le point de concours de fe et dc s'éloigne du point de concours de ba et 
dc. En effet, E'O et F'O interceptant le tableau curviligne IJ sous des angles plus extérieurs, le segment N'M est plus grand que le segment NM. La construction géométrique suggère une solution $x=0$ correspondant au cas des arêtes $\mathrm{CD}$ et EF confondues.

Supposons que la profondeur $y=\mathrm{AB}$ soit variable (Figure 5). Lorsque la fronto-horizontale BF s'éloigne de AE, le point de concours des fuyantes fe et dc s'éloigne du point de concours de ba et dc. Le segment N'M' est plus grand que le segment NM. La construction géométrique suggère la solution $y=0$, les arêtes $\mathrm{AE}$ et $\mathrm{BF}$ étant confondues.

Supposons enfin que la distance du spectateur à l'objet $z=\mathrm{BO}$ soit variable (Figure 6). On note que, à mesure que $\mathrm{BO}$ augmente, le rayon du cercle de projection IJ croît. Les points les plus extérieurs E et F découpent des angles plus petits, d'où N'M' > NM. La construction géométrique suggère une solution $z<0$, correspondant au cas où le point $\mathrm{O}$ divise le segment $\mathrm{AB}$ en deux parties égales. La condition initiale est alors vérifiée par raison de symétrie.

Ces solutions intuitives sont-elles les seules? Pour le savoir, reprenons la condition [14] et élevons tous les membres au carré :

[16] $\frac{\mathrm{OE}^{2}}{\mathrm{OC}^{2}}=\frac{\mathrm{OF}^{2}}{\mathrm{OD}^{2}}$

Le théorème de Pythagore donne :

[17] $\mathrm{OE}^{2}=(1+x)^{2}+(y+z)^{2}$

$$
\begin{aligned}
& \mathrm{OF}^{2}=(1+x)^{2}+z^{2} \\
& \mathrm{OC}^{2}=1+(y+z)^{2} \\
& \mathrm{OD}^{2}=1+z^{2}
\end{aligned}
$$

Les fuyantes d'une perspective synthétique admettent donc un point de concours pour les valeurs de $x, y, z$ vérifiant l'égalité :

[18] $\frac{(1+x)^{2}+(y+z)^{2}}{1+(y+z)^{2}}=\frac{(1+x)^{2}+z^{2}}{1+z^{2}}$

Soit : 
[19] $\left[1+z^{2}\right]\left[(1+x)^{2}+(y+z)^{2}\right]=\left[1+(y+z)^{2}\right]\left[(1+x)^{2}+z^{2}\right]$

On développe et on simplifie :

[20] $(1+x)^{2}+(y+z)^{2}+(1+x)^{2} z^{2}+(y+z)^{2} z^{2}=(1+x)^{2}+z^{2}+(1+x)^{2}(y+z)^{2}+(y+z)^{2} z^{2}$

$[21](y+z)^{2}+(1+x)^{2} z^{2}=z^{2}+(1+x)^{2}(y+z)^{2}$

$$
\begin{aligned}
& y^{2}+z^{2}+2 y z+z^{2}+x^{2} z^{2}+2 x z^{2}= \\
& z^{2}+y^{2}+z^{2}+2 y z+x^{2} y^{2}+x^{2} z^{2}+2 x^{2} y z+2 x y^{2}+2 x z^{2}+4 x y z
\end{aligned}
$$

On trouve alors l'équation quadratique [14 $\equiv 23]$ :

[23] $x^{2} y^{2}+2 x^{2} y z+2 x y^{2}+4 x y z=0$

dans laquelle on peut mettre $x$ et $y$ en facteurs :

$$
x y(x y+2 y+2 z(x+2))=0
$$

Il est aisé de constater que les seules solutions de cette équation sont:

[25] $x=0$ ou $y=0$ ou $z=-\frac{y}{2}$

$x=0$. Cette solution est incongrue car elle conduit à amputer l'objet de la travée CE et, par suite, à supprimer une fuyante. Une des conditions initiales - celle imposant l'examen d'au moins trois fuyantes - est alors violée.

$y=0$. Cette solution est incongrue car, A et B étant confondus, l'objet est sans profondeur. Dans ces conditions, l'angle d'une fuyante n'est plus déterminé et le point de concours peut être fixé arbitrairement.

$z=-\frac{y}{2} \Rightarrow z<0$. Cette solution est également incongrue car, lorsque le spectateur pénètre dans l'objet $(z<0)$, il fait face à ACE et ne voit plus la partie arrière de l'objet. En ce cas, le portique BDF n'est plus perçu. La condition [14] est alors dénuée de sens. 


\section{Conclusion}

Une perspective synthétique ne remplissant pas la condition [14] dans le cas général et les solutions particulières $x=0, y=0$ et $z<0$ étant incongrues, il n'existe par conséquent aucune solution au problème du parallélisme ou de la convergence stricte des fuyantes, ce qui réfute la conjecture Panofsky-White. ${ }^{24}$ Une perspective synthétique montre seulement une convergence approchée des fuyantes (qui est semblable à la section de la caustique produite par l'aberration de sphéricité en optique). On peut alors énoncer les théorèmes suivants :

Théorème 1 : Dans une perspective synthétique, les fuyantes n'admettent jamais de point de concours et ne sont jamais parallèles entre elles.

Réciproquement :

Théorème 2 : Toute représentation, dont trois fuyantes au moins sont parallèles ou admettent un point de concours, n'est pas une perspective synthétique.

Examinons les conséquences des ces propriétés pour la représentation perspective :

Corollaire 1 : Aucun parallélisme ni aucun point focal ne peut guider le peintre dans la construction d'une perspective synthétique : elle doit être construite à partir du plan géométral. (À l'inverse, une perspective ayant un point de fuite, ou répondant au parallélisme des fuyantes, peut avoir été construite sans géométral.)

Compte tenu du tracé géométrique des oeuvres étudiées (cf. Planches 1 et 2), ce corollaire indique que les artistes du Moyen Âge ont pu dessiner directement dans le plan du tableau sans s'aider de géométraux. Cela ne remet toutefois en question que l'usage perspectif des géométraux, dont les médiévaux pouvaient connaître l'existence à partir du traité de Vitruve, qui présente les différences entre ichnographia (plan), orthographia (élévation) et scaenographia (perspective). ${ }^{25}$ L'Élévation pour le campanile de Florence de Giotto, datée de 1334 (Sienne, Museo dell'Opera), qui est un exemple de géométral, ne prouve pas que Giotto ait utilisé de tels dessins auxiliaires pour tracer des perspectives.

Corrolaire 2 : la réfutation de la conjecture Panofsky-White valant pour les deux cas de convergence et de parallélisme, il n'y a plus de raison de penser à un rapport de parenté entre ces constructions. Il faut réinterpréter séparément les perspectives parallèles et les perspectives à deux points de fuite. 
Parallélisme des fuyantes. On peut, dans le cas du parallélisme des fuyantes, indiquer une construction beaucoup plus simple que celle de la perspective synthétique. Partons du fait que certaines représentations du Moyen Âge sont des perspectives cavalières (cf. L'Extase de Giotto, Figure 7, A). En faisant pivoter la face supérieure de l'objet sur l'arête horizontale postérieure, on obtient une perspective cavalière éclatée (cf. La Mort du chevalier de Celano, Figure 7, B). De même, on obtient une perspective cavalière doublement éclatée, en faisant pivoter la face latérale gauche de l'objet sur son arête verticale postérieure (cf. La Guérison du blessé de Léride, Figure 7, G). Le résultat de ces déformations est alors une boîte dont on peut voir les faces intérieures, comme c'est le cas du Retable du Maître de Sainte Cécile (Planche 1). Aucune de ces constructions ne suppose l'usage d'un géométral : elles se construisent directement dans le plan du tableau.

Convergence des fuyantes. Les oeuvres du corpus mentionnées à la note 21 montrent, à l'instar de Jésus parmi les Docteurs de Giusto de’ Menabuoi (Planche 2), la convergence des fuyantes en deux points de fuite. Elles ne peuvent pas porter le nom de «perspective bifocale » réservé aux perspectives linéaires utilisant des points de distance (ce serait le cas, par exemple, d'un cube dont aucune face ne serait perpendiculaire à l'axe de vision OK). Les deux points que l'on observe sur les oeuvres du corpus correspondent en réalité à deux « points de fuite centraux » sans rapport avec la perspective bifocale. Il a été avancé que ces constructions - hétérodoxes, au vu des conventions adoptées à la Renaissance - ont pu résulter d'une application qualitative des principes de la vision binoculaire. ${ }^{26}$ Ce système de représentation s'expliquerait ainsi : la distance entre le cadre architectural (à l'arrière-plan) et le sujet principal de la scène (au premier plan) sur lequel se fait la fixation occasionne une « diplopie homonyme », c'est-àdire une vision double de l'objet situé à l'arrière-plan. ${ }^{27}$ Cette diplopie cause le dédoublement des points de fuite car le cadre architectural, dont les arêtes rectilignes donnent les fuyantes de la perspective, se trouve en arrière du personnage central sur lequel s'opère la fixation. Les deux points focaux résultent alors de la disparité des quasi-images produites par l'oeil gauche et par l'oeil droit. ${ }^{28}$

La vraisemblance de cette hypothèse tient au fait que les principaux traités d'optique du Moyen Âge - Ibn al-Haytham, ${ }^{29}$ Kitâb al-manâzir (De aspectibus), III, 12 ; Bacon, ${ }^{30}$ Perspectiva, II, II, 1 -3 ; Pecham, ${ }^{31}$ Perspectiva communis, I, 32 ; Witelo, ${ }^{32}$ Perspectiva, III, 27 sq. - présentent des caractéristiques à même de justifier cette application : 1) ces textes traitent en détail des problèmes de la vision binoculaire sans jamais introduire la théorie de la neutralisation (qui 
annulerait la diplopie) ; 2) ces traités d'optique étaient connus en Italie à l'époque où ces perspectives ont été réalisées, et dans les milieux mêmes où elles ont été réalisées ${ }^{33}$; 3) Vitruve, beaucoup lu au Moyen Âge, recommandait aux architectes d'étudier l'optique, ${ }^{34}$ pratique dont il nous reste d'ailleurs quelques témoignages isolés. ${ }^{35}$ 

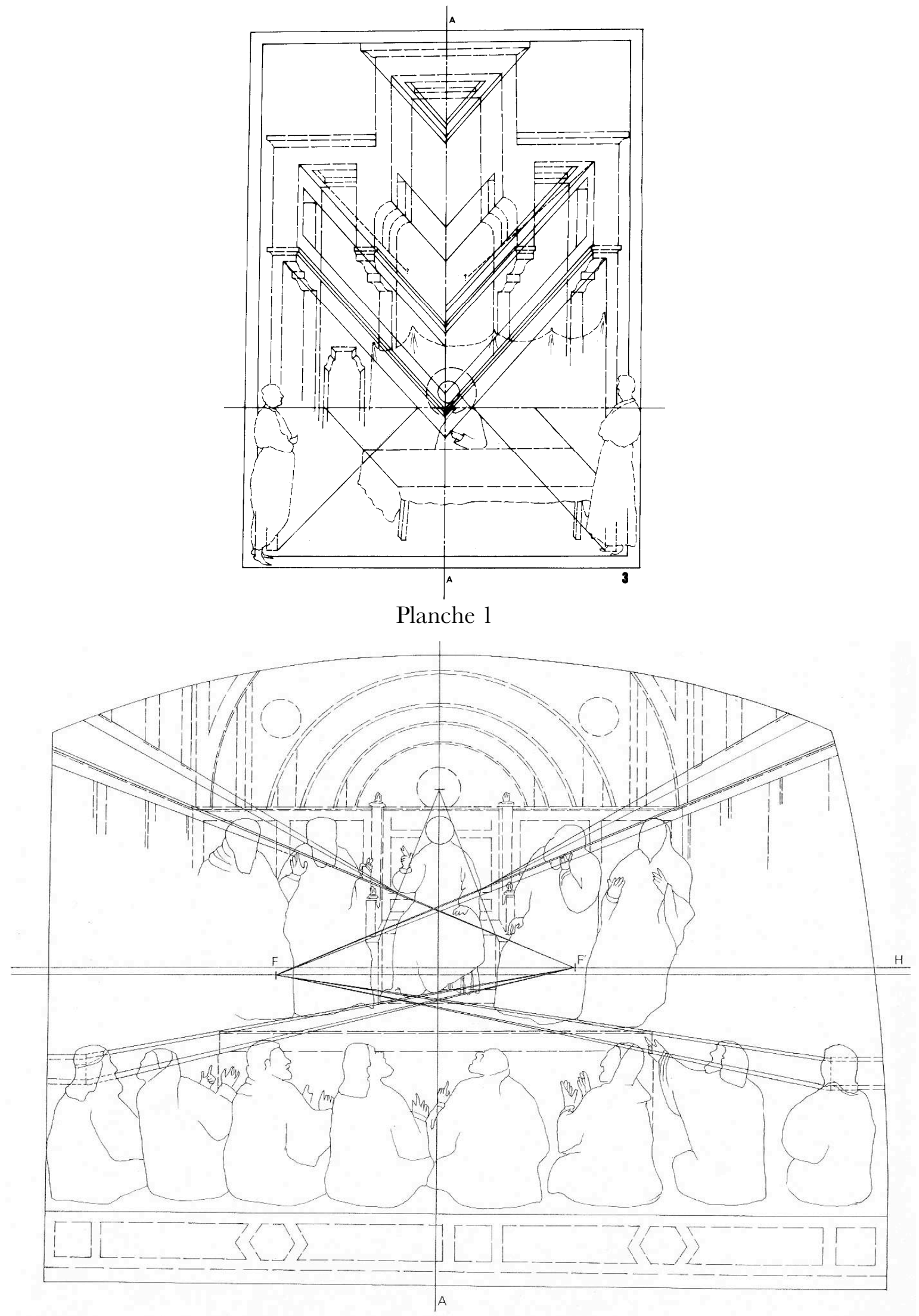

Planche 2 
${ }^{1}$ Université Pierre-Mendès-France, BP 47, 38040 Grenoble cedex 9, Laboratoire d'architecturologie et de recherches épistémologiques sur l'architecture (Paris), dominique.raynaud@upmf-grenoble.fr.Je remercie Mohamed el-Methni (LabSAD, Grenoble) qui a su distinguer la propriété [14] et m'a fait part d'utiles remarques sur une version préparatoire du manuscrit.

2 Cf. Giuseppe Basile, Giotto. Le storie francescane. Milano 1996.

${ }_{3}^{3}$ Nous suivrons avec réticence l'usage consistant à parler de « fuyantes », de « point de fuite », d'« horizon », etc. Il vaudrait mieux employer des néologismes parce que ces notions n’ont pris de signification mathématique qu'à partir du XVIIe siècle (Guidobaldo del Monte, Desargues). Il est difficile de connaître le sens que les artistes du Moyen Âge attribuaient aux lignes et points de construction. Dans le cadre de cet article, les concepts perspectifs ont simplement une fonction d'indexation des figures: ils sont privés de toute fonction sémantique.

${ }^{4}$ Guido J. Kern, Die Anfänge der zentralperspektivischen Konstruktion in der italianischen Malerei des 14. Jahrunderts, in: Mitteilungen des Kunsthistorischen Instituts in Florenz 2, 1913, 39-65.

${ }^{5}$ Erwin Panofsky, La Perspective comme "forme symbolique" et autres essais. Paris 1975 (1e éd. 1924).

${ }^{6}$ Panofsky (note 5), p. 122 (mes italiques).

7 «Item scaenographia est frontis et laterum abscedentium adumbratio ad circinique centrum omnium l inearum responsus », De Architectura, I, I, cf. Frank Granger, Vitruvius on architecture. 2 vols. Cambridge 1956, vol. 1, p. 26.

${ }^{8}$ Hermann von Helmholtz et Ernst W. von Brücke, Principes scientifiques des beaux-arts, essais et fragments de thé orie. L'optique et la peinture. Paris 1878; Guido Hauck, Die subjektive Perspektive und die horizontalen Curvaturen des dorischen Styls. Stuttgart 1879.

${ }_{9}^{9}$ Alan M. G. Little, Scaenographia, in: The Art Bulletin 18, 1936, 407-418, Idem, Perspective and scene pai nting, in: The Art Bulletin 20, 1937, 487-495, Miriam S. Bunim, Space in Medieval Painting and the Forerunners of Perspective. New York 1940, John White, Perspective in Ancient Drawing and Painting. London 1956, Idem, The Birth and Rebirth of Pictorial Space. London 1967. Voir aussi les analyses pénétrantes de Gezienus ten Doesschate, Perspective. Fundamentals, Controversials, History. Nieuwkoop 1964, p. 85-99 et 105-118.

10 Little (note 9).

${ }^{11}$ Bunim (note 9), reconnaît que, si un peintre comme « Simone Martini a utilisé la procédure de l'axe de fuite », « Dans les peintures de Giotto, la procédure de l'axe de fuite pour les plans verticaux en profondeur ne suit pas une forme systématique clairement définie de droites de bout convergeant deux à deux » (p. 141, 143). Mais de nombreux cas en faveur de la théorie de l'axe de fuite sont ensuite donnés à propos de Simone Martini et Barna da Siena (p. 148) et de la peinture italienne du XIVe siècle (p. 154, 157, 166 et 174).

${ }^{12}$ Bunim (note 9), p. 24-25.

13 White (note 9).

14 White 1967 (note 9), p. 213.

15 André Flocon et André Barre， La perspective curviligne. De l'espace visuel à l'image construite . Paris 1968. Ces principes auraient été mis en oeuvre par Jean Fouquet, Entrée de l'empereur Charles IV à Saint-Denis, 1460 (Paris, 
Bibliothèque Nationale, ms. fr. 247, fol. 163). Quant au fondement géométrique de cette construction, nous en sommes réduits aux conjectures.

${ }^{16}$ Panofsky (note 5), Little, Bunim et White (note 9).

${ }_{17}$ Hendrick G. Beyen, Die pompejanische Wanddekoration (vom zweiten bis zum vierten Stil) . 2 vols., Haag 1938, Idem, Die antike Zentralperspecktive, in: Jahrbuch des deutschen archäologischen Instituts 54, 1939, 47-72, Decio Gioseffi, Perspectiva artificialis. Per la storia della prospettiva, spigolature e appunti. Trieste 1957. Gezienus ten Doesschate, Perspective. Fundamentals, Controversials, History. Nieuwkoop 1964. Luigi Vagnetti, De naturali et artificiali perspectiva, in: Studi e Documenti di Architettura 9/10, 1979, 3-520. Gérard Simon, Optique et perspective: Ptolémée, Alhazen, Alberti, in: Revue d'Histoire des Sciences 54, 2001, 325-350.

${ }^{18}$ Andrés de Mesa Gisbert, El 'fantasma' del punto de fuga en los estudios sobre la sistematización geométr ica de la pintura del siglo XIV, in: D’Art 15, 1989, 29-50, p. 49.

${ }^{19}$ Antonella Ballardini, Lo spazio pittorico medievale: Studi e prospettive di ricerca, in: Rocco Sinisgalli, ed., La prospettiva. Fondamenti teorici ed esperienze figurative dall'Antichità al mondo moderno, Convegno Internazionale di Studi (Roma, 11-14 settembre 1995). Firenze 1998, 281-292, p. 281. De même que celles de Panofsky, les vues de White ont été très largement diffusées, The Birth and Rebirth ayant connu quatre éditions en langue anglaise (1967, 1970, 1972, 1987) et ayant été traduit en plusieurs langues (dont l'édition française de 1992).

${ }^{20}$ Panofsky (note 5), p. 76.

${ }^{21}$ Les conclusions de l'analyse empirique des perspectives sont dépendantes des erreurs de tracé . Voici la méthodologie sur laquelle nous nous sommes appuyés. Nous avons réuni un corpus d'une cinquantaine d'œuvres répondant au type de la construction axiale. Nous en avons exclu les tableaux dont le cadre architectural présentait moins de trois fuyantes issues du même côté du tableau (deux fuyantes ayant toujours un point de concours) et les tableaux présentant des erreurs accidentelles importantes. De la sorte, le corpus a été réduit à trente oeuvres réalisées en Italie centrale entre 1295 et 1450. De manière à obtenir des conclusions nettes, nous avons procédé au contrôle des erreurs de reconstruction selon le protocole présenté in Dominique Raynaud, La théorie des erreurs et son application à la reconstruction des tracés perspectifs, in: Marisa Dalai, Pascal Dubourg Glatigny, Marianne Le Blanc, eds., L'artiste et l'oeuvre à l'épreuve de la perspective, Colloque international (Rome, 1921 septembre 2002). Rome (sous presse). Nous donnons à la suite la liste des œuvres examinées [K coefficient de réduction, FF' distance entre les points de fuite $(\mathrm{cm}), \varepsilon$ erreur de reconstruction $(\mathrm{cm}), \mathrm{FF}^{\prime} / \varepsilon$ rapport distance/erreur]:

$\mathrm{n}^{\circ}$ Auteur, Titre de l'oeuvre (date)

1. Maître d'Isaac, La Pentecôte (c. 1295)

2. Giotto, L'Approbation de la règle franciscaine (1296-99)

3. Giotto, Le Prêche devant Honorius III (1296-99)

4. Giotto, Jésus devant Anas et Caïphe (1304-06)

5. Giotto, Vierge en majesté (c. 1306)

6. Giotto, Fusticia (c. 1308)

$\begin{array}{rrrr}\mathrm{K} & \mathrm{FF} & \varepsilon & \mathrm{FF}^{\prime} / \varepsilon \\ 15,0 & 15 & 1,3 & 11,5 \\ 12,8 & 179 & 1,7 & 105,3 \\ 12,0 & 16 & 0,7 & 22,8 \\ 9,3 & 140 & 1,2 & 116,7 \\ 15,9 & 72 & 2,1 & 34,3 \\ 5,2 & 117 & 1,5 & 78,0\end{array}$


7. Duccio di Buoninsegna, Les Noces de Cana (1308-11)

\begin{tabular}{|c|c|c|c|}
\hline 2,2 & 72 & 2,0 & 36,0 \\
\hline 2,5 & 71 & 2,0 & 35,5 \\
\hline 3,9 & 76 & 1,6 & 47,5 \\
\hline 15,5 & 6 & 1,6 & 3,7 \\
\hline 14,6 & 19 & 1,7 & 11,2 \\
\hline 14,3 & 4 & 1,0 & 4,0 \\
\hline 2,4 & 73 & 1,7 & 43,7 \\
\hline 7,9 & 7 & 0,7 & 10,0 \\
\hline c. 4 & 67 & 2,9 & 23,1 \\
\hline 17,2 & 28 & 0,9 & 31,1 \\
\hline c. 5 & 35 & 1,1 & 31,8 \\
\hline 5,7 & 20 & 0,8 & 25,0 \\
\hline 7,0 & 31 & 1,01 & 30,7 \\
\hline c. 10 & 52 & 2,3 & 22,6 \\
\hline 14,1 & 77 & 1,0 & 77,0 \\
\hline 5,0 & 31 & 1,3 & 23,8 \\
\hline 1,8 & 58 & 1,2 & 48,3 \\
\hline 1,9 & 111 & 1,8 & 61,7 \\
\hline 1,7 & 31 & 1,0 & 31,0 \\
\hline 4,6 & 116 & 0,7 & 165,7 \\
\hline 1,7 & 143 & 0,7 & 204,3 \\
\hline 1,7 & 104 & 1,1 & 94,5 \\
\hline 5,7 & 19 & 2,1 & 9,0 \\
\hline 1,6 & 6 & 1,3 & \\
\hline
\end{tabular}

8. Duccio, L'Apparition du Christ par une porte close (1308-11)

9. Duccio, La Dernière Cène (1308-11)

10. Simone Martini, La Mort de Saint François (1315-17)

11. Simone Martini, Les Funérailles de Saint François (1315-17)

12. Maestro senesegiante, Fésus parmi les Docteurs (1315-1320)

13. Giotto, Madone et saints (1328)

14. Pietro Lorenzetti, La Nativité (1342)

15. Giusto de' Menabuoi, Madone sur le trône (1349)

16. Barna da Siena, Le Pacte de Fudas (c. 1350)

17. Tommaso da Modena, Saint Romuald (1352)

18. Lorenzo Veneziano, Madone à l'enfant (1372)

19. Giusto de' Menabuoi, Saint sur le trône (c. 1370)

20. Altichiero, Le Conseil du Roi Ramire (c. 1374-79)

21. Giusto de' Menabuoi, Jésus parmi les Docteurs (1376-78)

22. Stefano di Sant'Agnese, Madone à l'enfant (c. 1390)

23. Taddeo di Bartolo, La Cène (1394-01)

24. Lorenzo Monaco, L'Adoration des Mages (c. 1421)

25. Lorenzo Ghiberti, Le Christ parmi les Docteurs (c. 1415)

26. Niccolò di Pietro, Saint Benô̂t exorcisant un moine (c. 1420)

27. Gentile da Fabriano, Infirmes au tombeau de Saint Nicolas (1425)

28. Giovanni di Ugolino, Madone à l'enfant (1436)

29. Donatello, La Confession du nouveau-né (1447-48)

30. Fra Angelico, Le Christ bafoué (1450)

${ }^{22}$ Kern (note 4).

${ }^{23} \mathrm{Il}$ suffit de fixer les mesures suivantes: $\mathrm{AC}=1, \mathrm{CE}=1, \mathrm{AB}=1, \mathrm{OB}=3$. En ce cas, on devrait avoir: $\frac{(\mathrm{AC}+\mathrm{CE})^{2}+(\mathrm{OB}+\mathrm{BA})^{2}}{(\mathrm{AC})^{2}+(\mathrm{OB}+\mathrm{BA})^{2}}=\frac{(\mathrm{AC}+\mathrm{CE})^{2}+(\mathrm{OB})^{2}}{(\mathrm{AC})^{2}+(\mathrm{OB})^{2}}$ or $\frac{20}{17} \neq \frac{13}{10}$

${ }^{24}$ Les principes de la perspective synthétique n’ont donc pas été connus au Moyen Âge et il est peu probable qu'ils aient été appliqués avant le XIX siècle, cf. Arthur Parsey, Perspective rectified. London 1836, William G. Herdman, A Treatise on the Curvilinear Perspective of Nature. London 1853, Guido Hauck, Die subjektive Perspektive und die horizontalen Curvaturen des dorischen Styls. Stuttgart 1879.

25 « Ichnographia est circini regulaeque modice continens usus, e qua capiuntur formarum in solis arearum descriptiones. Orthographia autem est erecta frontis imago modiceque picta rationibus operis futuri figura. Item 
scaenographia est frontis et laterum abscendentium adumbratio ad circinique centrum omnium linearum responsus », De Architectura, I, II, cf. Granger (note 7), vol. 1, p. 24-26.

${ }^{26}$ Dominique Raynaud, Perspective curviligne et vision binoculaire, in: Sciences et Techniques en Perspective 2, 1998, 3-23, Idem, Ibn al-Haytham sur la vision binoculaire: un précurseur de l'optique physiologique, in: Arabic Sciences and Philosophy 13, 2003, 79-100.

${ }^{27}$ Hermann von Helmholtz, Optique physiologique. Paris 1867, Yves Le Grand, Optique physiologique, 3 vols. Paris 1948-1956.

${ }^{28}$ On peut en acquérir la certitude par une expérience simple. Plaçons-nous à quelques mètres d'une bougie et tendons l'index dans la direction de la bougie. Si l'on fixe la bougie, l'index est vu double (diplopie croisée), si l'on fixe l'index, la bougie est vue double (diplopie homonyme). On trouvera une analyse de quelques textes médiévaux sur la vision binoculaire et leur application à la perspective in: Raynaud (note 26).

${ }^{29}$ Abdelhamid I. Sabra, The Optics of Ibn al-Haytham, Books I-III: On Direct Vision. London 1989.

${ }^{30}$ John H. Bridges, The Opus Maius of Roger Bacon . Frankfurt am Main 1964, David C. Lindberg, Roger Bacon and the Origins of Perspectiva in the Middle Ages. Oxford 1996.

${ }^{31}$ David C. Lindberg, Vitellonis Thuringopoloni opticae libri decem. New York/London 1972.

32 David C. Lindberg, Fohn Pecham and the Science of Optics: Perspectiva Communis. Madison 1970.

${ }_{33}$ Cf. notamment Luca Baggio, Sperimentazioni prospettiche e ricerche scientifiche a Padova nel secondo Trecento, in: Il Santo 34, 1994, 173-232, Francesca Cecchini, Artisti, committenti e perspectiva in Italia alla fine del Duecento, in: Rocco Sinisgalli, ed., La prospettiva. Fondamenti teorici ed esperienze figurative dall'Antichità al mondo moderno, Convegno Internazionale (Roma, 11-14 settembre 1995). Firenze 1998, 56-74 et Dominique Raynaud, L'Hypothèse d'Oxford. Essai sur les origines de la perspective. Paris 1998.

${ }^{34}$ Vitruve écrit par exemple: «Item per opticen in aedificis $[\ldots] »$, « $[\ldots]$ a geometris de visu qui graece 1 ogos opticos appellatur », De architectura, I, I, cf. Granger (note 7), vol. 1, p. 8, 20.

${ }^{35}$ La fin de l'épigraphe de Pistoia nous apprend que Giovanni Pisano connaissait l'optique: « Fohannes qui res non egit inanes Nicoli natus sensia meliores beatus quem genuit Pisa doctum super omnia visa »(mes italiques). 